\title{
Study on Influence Factors of Pressure Injury Risk in the Elderly Inpatients with Kidney Disease Based on LASSO Regression
}

\author{
Ling Liu\#, Chunhua Wang\#, Lianghong Yin, Jiayi Wang, Hong Yang, \\ Yingxue Zhong*, Zhiwei Mou*, Yu Chen* \\ Nephrology Department, The First Affiliated Hospital of Jinan University, Guangzhou, China \\ Email: ^yxz1108@126.com, *mouzhiwei@jnu.edu.cn, ${ }^{\star}$ yusichen@jnu.edu.cn
}

How to cite this paper: Liu, L., Wang, C.H., Yin, L.H., Wang, J.Y., Yang, H., Zhong, Y.X., Mou, Z.W. and Chen, Y. (2020) Study on Influence Factors of Pressure Injury Risk in the Elderly Inpatients with Kidney Disease Based on LASSO Regression. Open Journal of Preventive Medicine, 10, 95-107.

https://doi.org/10.4236/ojpm.2020.106007

Received: May 7, 2020

Accepted: June 5, 2020

Published: June 8, 2020

Copyright $\odot 2020$ by author(s) and Scientific Research Publishing Inc. This work is licensed under the Creative Commons Attribution International License (CC BY 4.0).

http://creativecommons.org/licenses/by/4.0/

(c) (i) Open Access

\begin{abstract}
Objective: This paper aims to explore clinical status and related influence factors of pressure injury (PI) in the elderly inpatients with kidney disease, so as to provide reference for the prevention and treatment of PI in the elderly inpatients with kidney disease. Methods: Retrospective collection method is adopted to collect 158 clinical cases of the elderly inpatients with kidney disease aged $\geq 60$ in the Nephrology Department, the First Affiliated Hospital of Jinan University from January 2017 to December 2019, and then least absolute shrinkage and selection Operator (LASSO) regression analysis is used to analyze 17 possible influence factors; finally Logistic regression model is established to analyze and screen influence factors of risk. Results: 1) Among 158 elderly inpatients with medium and high risk of PI, the incidence of PI is $20.25 \%$; the most common stage of injury is stage I (42.5\%); sacrococcygeal (60\%) is the high-risk site of pressure injury. 2) LASSO regression analysis shows that history of present respiratory infection/respiratory failure $(\beta=1.2714 . P<0.05)$ and hospitalization time $(\beta=0.4177 . P<0.05)$ are independent factors influencing PI risk in the elderly inpatients with kidney disease. Conclusion: The elderly patients with kidney disease and PI risk are the high incidence population of hospital acquired PI; for the elderly inpatients with kidney disease and having respiratory infection history or respiratory failure, prolonged hospitalization will significantly increase the risk of PI. Therefore, targeted preventive and control measures should be taken to reduce the incidence of PI.
\end{abstract}

\section{Keywords}

Least Absolute Shrinkage and Selection Operator, The Elderly Inpatients with Kidney Disease, Pressure Injury, Influence Factors, Nursing

\#These authors contributed to the work equally and should be regarded as co-first authors.

*These authors are co-corresponding authors. 


\section{Introduction}

In 2016, the National Pressure Ulcer Advisory Panel (NPUAP) updated the definition of pressure ulcer and changed the name of "pressure ulcer" to "pressure injury". Pressure injury refers to limited injury of skin and (or) subcutaneous soft tissue, usually located in the bone bulge, or related to medical devices. Relatively speaking, the use of the term "injury" covers a wider range, including both open ulcer and tissue injury in deep positions of the body without obvious appearance [1]. At the same time, pressure injury related to medical devices and mucosal pressure injury are also included in the scope of pressure injury.

Pressure injury (PI) is a common chronic refractory wound in clinic. Recent studies have shown that the incidence and prevalence rates of PI have remained high [2]. Another study shows that with age growing, the incidence of PI is also increasing, and more than $70 \%$ of PI patients are over 70 years old [3]. In 1999, China entered the aging society, the incidence of various acute and chronic diseases of the elderly increased significantly. Kidney disease has become the third major disease affecting the health of the elderly in China [4]. The elderly patients with kidney disease have low immunity, which often leads to various concurrent infections. Once pressure injury occurs, the hospitalization time will increase; and the elderly patients will be seriously infected, which will make them feel worse and even threaten their life [5]. At the same time, the workload of medical staff increases, and the satisfaction of patients and their families decreases. The incidence of hospital acquired PI is one of the key indicators to evaluate nursing quality [6]. Therefore, it is one of the key and difficult points to prevent and reduce the complication of PI in the elderly inpatients with kidney disease. At present, there are few evidence-based studies on the clinical practice guidance of PI prevention and treatment, and the prevention and treatment guidance of PI in the elderly patients with kidney disease has not been retrieved. This study reviews the elderly patients with kidney disease and with medium and high risk of PI in three years of undergraduate course, and uses LASSO Logistic regression model to analyze and screen the relevant influence factors of PI occurrence, so as to provide reference for clinical researches on the improvement of PI prevention measures and reduction of PI incidence.

\section{Research Subjects and Methods}

\subsection{Research Subjects}

From January 2017 to December 2019, among cases of the elderly inpatients with kidney disease who had PI in the Nephrology Department of our hospital, 32 cases were included in this study. Inclusion criteria: 1) Meeting the definition of PI of NPUAP in 2016;2) Age $\geq 60$; 3) Hospitalization time in Nephrology Department $>24$ hours; 4) Norton scale score $\leq 14$ points. Exclusion criteria: 1) Patients with pressure injury on admission; 2) patients with extremely critical conditions and patients who are forbidden to turn their bodies; 3) patients with 
Burn or acute or chronic skin diseases. In non-PI group, 126 elderly inpatients with kidney disease who were admitted to the Nephrology Department in the same year and did not have PI during hospitalization were collected by a continuous enrollment method.

\subsection{Research Methods}

\subsubsection{Questionnaire of the Elderly Patients with Kidney Disease and High-Risk PI}

By using literature research method and investigation \& discussion method, the researcher designed the questionnaire of factors related to PI in hospital. The questionnaire includes the following contents: 1) Basic information: name, gender and age. 2) Possible factors related to PI in hospital: main diagnosis, hospitalization time, maximum body temperature, complications (hypertension, diabetes, coronary heart disease/heart failure, respiratory infection)/respiratory failure and craniocerebral disease, etc.), edema, limb activity (independent, partially dependent or completely dependent), urination (controllable, occasional incontinence, complete incontinence or need other people's catheterization), Norton scale score, laboratory examination indicators (first serum albumin detection on admission, hemoglobin, blood creatinine, etc.). 3) PI occurrence: quantity, position and stage.

\subsubsection{Index Evaluation Method}

1) Norton scale: the first form from the study of Gerontology in 1963, which is generally applicable to elderly wards. The assessment form includes 5 items: general physical condition, mental condition, walking ability, activity ability and incontinence. The score ranges from 5 to 20, 15 - 19 for mild risk, 13 - 14 for moderate risk, and $\leq 12$ for high risk. The lower the score is, the higher the probability of pressure injury occurs [7] [8]. Professor Rabinovitz's research on Norton scale in the UK showed: Norton scale could be used as the first preferred evaluation tool in the prevention of pressure injury in the elderly [9]. A domestic study using Norton scale to test the elderly patients also confirmed that Norton scale, especially in the prevention of patients with kidney disease and high-risk pressure injury, was more targeted, and it could also enhance the awareness of nurses to take the initiative to prevent pressure injury [10].

2) PI staging: according to NPUAP [1] in 2016, in PI staging system, Roman numerals (I, II, III, IV) are replaced by Arabic numerals (1, 2, 3, 4), in addition to non-staged pressure injury and tissue pressure injury in deep positions.

3) PI record table: it is used to record the wound conditions of patients with current or previous PI, including PI stages, location, size, treatment measures, wound healing, etc.

\subsubsection{Data Collection Method}

The data collection of cases was completed by 2 nurses in the investigation group who had more than 5 years' clinical nursing time. Before the formal investigation, the main researcher conducted a group training on the objectives, contents, methods and precautions of the investigation, collecting data according to 
the unified standard. Methods of data collection: the 2 nurses comprehensively used the hospital electronic integration system to look up inpatients' medical records of the subjects by retrospective analysis, including general information of inpatients, Norton scale score, combined diseases, related laboratory examination indicators and other 17 possible risk factors. The collected data was checked by the third researcher in the investigation group and then input into Excel software to ensure the authenticity of the data.

\subsubsection{Basic Principles and Advantages of LASSO Logistic Regression Model}

In 1996, Robert Tibshirani proposed a new variable selection technique LASSO, which was characterized by variable selection and complexity adjustment while fitting the generalized linear model [11]. The basic idea of LASSO regression is to minimize the sum of the squared residuals when the sum of absolute values of a regression coefficient is less than a constant, and then strictly compresses the unimportant regression coefficient to zero and eliminate it, so as to realize variable selection [12]. LASSO Logistic regression model is established by selecting variables through LASSO method.

\subsection{Statistical Analysis}

EPI data 3.1 was used for data entry and R 3.6.2 was used for statistical analysis. LASSO regression analysis was applied to the potential PI related factors. When selecting the optimal Lambda value [13], Logistic regression model was used to do analyzing and screening, and two related prognostic parameters were included to further construct the nomogram. $P<0.05$ was statistically significant.

\section{Results}

\subsection{Basic Information of Research Subjects}

In this study, 158 the elderly inpatients with kidney disease were enrolled, including 32 cases in PI group and 126 cases in non-PI group. The age of PI group was $73.8 \pm 7.59,19(59.38 \%)$ men and 13 (40.62\%) women; Norton risk score was $(11 \pm 2.66)$; the age of non-PI group was $75.3 \pm 7.99$ : $52(41.27 \%)$ men and $74(58.73 \%)$ women; Norton risk score was $(12.09 \pm 2.09)$. The clinical characteristics of different groups are shown in Table 1.

\subsection{Incidence of PI in the Elderly Inpatients with Kidney Disease}

The cases of hospital acquired PI patients were 32, its incidence being 20.25\% (32/158). PI staging: 17 cases (42.5\%) in the first stage, 14 cases (35\%) in the second stage, 4 cases (10\%) in the third stage and 5 cases $(12.5 \%)$ in the deep tissue injury stage. The hospital acquired PI occurred 40 times, one time in each case of 26 cases $(81.25 \%)$, two times in each case of 5 cases (15.63\%), and four times in 1 case (3.13\%). Times of PI occurred in the location were 24 times in sacrococcygeal region (60\%), 6 times in ischial region (left/right), 4 times in heel region (10\%), 2 times in occipital region (5\%), 2 times in femoral tuberosity 
Table 1. Demographic and clinical characteristics of elderly inpatients with kidney disease (\%).

\begin{tabular}{|c|c|c|c|c|c|}
\hline Variables & $\begin{array}{l}\text { PI Group } \\
(n=32)\end{array}$ & $\begin{array}{l}\text { Non-PI Group } \\
\quad(n=126)\end{array}$ & Variables & $\begin{array}{l}\text { PI Group } \\
(n=32)\end{array}$ & $\begin{array}{l}\text { Non-PI Group } \\
\quad(n=126)\end{array}$ \\
\hline Gender & & & Edema & & \\
\hline $\mathbf{M}$ & $19(59.38 \%)$ & $52(41.27 \%)$ & No & $19(59.38 \%)$ & $93(73.81 \%)$ \\
\hline F & $13(40.62 \%)$ & $74(58.73 \%)$ & Yes & $13(40.63 \%)$ & $33(26.19 \%)$ \\
\hline Age(years old) & & & The highest body temperature & & \\
\hline $60 \sim$ & $12(37.5 \%)$ & $34(26.98 \%)$ & $\left({ }^{\circ} \mathrm{C}\right) \geq 37.3$ & & \\
\hline $70-79$ & $11(34.38 \%)$ & $47(37.30 \%)$ & No & $7(21.88 \%)$ & $42(33.33 \%)$ \\
\hline$\geq 80$ & $9(28.13 \%)$ & $45(35.71 \%)$ & Yes & $25(78.13 \%)$ & $84(66.67 \%)$ \\
\hline Hospitalization time(d) & & & Norton score (points) & & \\
\hline$>1$ & $0(0 \%)$ & $13(10.32 \%)$ & 13 - 14 points (medium risk) & $10(31.25 \%)$ & $69(54.76 \%)$ \\
\hline $8 \sim$ & $4(12.5 \%)$ & $29(23.02 \%)$ & $\leq 12$ points (high risk) & $22(68.75 \%)$ & $57(45.24 \%)$ \\
\hline $15 \sim$ & $6(18.75 \%)$ & $29(23.02 \%)$ & & & \\
\hline $22 \sim$ & $4(12.5 \%)$ & $17(13.49 \%)$ & & & \\
\hline$\geq 29$ & $18(56.25 \%)$ & $38(30.16 \%)$ & & & \\
\hline Main diagnosis & & & Limb mobility & & \\
\hline CKD5 stage & $28(87.5 \%)$ & $94(74.60 \%)$ & Independent & $0(0 \%)$ & $5(3.97 \%)$ \\
\hline \multirow[t]{2}{*}{ Non-CKD5 stage } & $4(12.5 \%)$ & $32(25.40 \%)$ & Partially dependent & $10(31.25 \%)$ & $63(50 \%)$ \\
\hline & & & Completely dependent & $22(68.75 \%)$ & $58(46.03 \%)$ \\
\hline Diabetes & & & Defecation & & \\
\hline No & $10(31.25 \%)$ & $60(47.62 \%)$ & Controllable & $7(21.88 \%)$ & $51(40.48 \%)$ \\
\hline \multirow[t]{2}{*}{ Yes } & $22(68.75 \%)$ & $66(52.38 \%)$ & Occasionally incontinent & $11(34.38 \%)$ & $47(37.30 \%)$ \\
\hline & & & Completely incontinent & $14(43.75 \%)$ & $28(22.22 \%)$ \\
\hline Hypertension & & & Serum albumin $A L B(g / L)$ & & \\
\hline No & $6(18.75 \%)$ & $22(17.46 \%)$ & $>35$ & $13(40.63 \%)$ & $44(34.92 \%)$ \\
\hline \multirow[t]{2}{*}{ Yes } & $26(81.25 \%)$ & $104(82.54 \%)$ & $30 \sim$ & $6(18.75 \%)$ & $43(34.13 \%)$ \\
\hline & & & $<30$ & $13(40.63 \%)$ & $39(30.95 \%)$ \\
\hline Coronary disease & & & Hemoglobin HGB (g/L) [14] & & \\
\hline No & $19(59.38 \%)$ & $71(56.35 \%)$ & Normal: >120 (M)/110 (F) & $4(12.5 \%)$ & $29(23.02 \%)$ \\
\hline Yes & $13(40.63 \%)$ & $55(43.65 \%)$ & Mild: 90 - $120(\mathrm{M}) / 110(\mathrm{~F})$ & $13(40.63 \%)$ & $37(29.37 \%)$ \\
\hline \multirow[b]{2}{*}{ Respiratory infection/respiratory failure } & & & Moderate: 60 - 90 & $8(25 \%)$ & $54(42.86 \%)$ \\
\hline & & & Severe: 30 - 59 & 7 (21.88\%) & $6(4.76 \%)$ \\
\hline No & $8(25 \%)$ & $74(58.73 \%)$ & \multirow[t]{2}{*}{ Extremely severe: $<30$} & \multirow[t]{2}{*}{$0(0 \%)$} & \multirow[t]{2}{*}{$0(0 \%)$} \\
\hline Yes & $24(75 \%)$ & $52(41.27 \%)$ & & & \\
\hline Craniocerebral diseases & & & Serum creatinine CREA & & \\
\hline No & $13(40.63 \%)$ & $77(61.11 \%)$ & $(\mathrm{umol} / \mathrm{L})[14]$ & & \\
\hline \multirow[t]{3}{*}{ Yes } & $19(59.38 \%)$ & $49(38.89 \%)$ & $<450$ & $18(56.25 \%)$ & $79(62.70 \%)$ \\
\hline & & & $450-707$ & $9(28.13 \%)$ & $31(24.60 \%)$ \\
\hline & & & $>707$ & $5(15.63 \%)$ & $16(12.70 \%)$ \\
\hline
\end{tabular}


region (5\%), and 2 times in ankle region (5\%). See Table 2.

\subsection{Multivariate Analysis of PI Risk in the Elderly Inpatients with Kidney Disease}

1) According to variable selection from LASSO regression, the results were: history of present respiratory infection/respiratory failure $(\beta=0.958)$, craniocerebral disease $(\beta=0.446)$, limb mobility $(\beta=0.387)$, diabetes mellitus $(\beta=$ $0.364)$, hospitalization time $(\beta=0.269)$, edema $(\beta=0.273)$, Norton score $(\beta=$ $0.266)$, defecation $(\beta=0.228)$, hemoglobin $(\beta=0.136)$, serum creatinine $(\beta=$ $0.093)$, hypertension $(\beta=-0.301)$, and gender $(\beta=-0.616)$. The meaning of each variable is shown in Table 3 below.

2) In this study, 17 potential PI risk factors were included, including basic information of subjects, clinical information, laboratory examination characteristics, etc. LASSO regression analysis of all the different factors showed that the

Table 2. Stages and locations of PI in the elderly inpatients with kidney disease [times (percentage, \%)].

\begin{tabular}{cccccccc}
\hline Stages Locations & First stage & Second stage & Third stage & Fourth stage & Indivisible & Deep tissue & Total \\
\hline Sacrococcygeal & $11(27.5 \%)$ & $9(22.5 \%)$ & $2(5 \%)$ & 0 & 0 & $2(5 \%)$ & $24(60 \%)$ \\
Ischium (left/right) & $1(2.5 \%)$ & $4(10 \%)$ & $1(2.5 \%)$ & 0 & 0 & 0 & $6(15 \%)$ \\
Heel & $1(2.5 \%)$ & 0 & $1(2.5 \%)$ & 0 & 0 & $2(5 \%)$ & $4(10 \%)$ \\
Occipital bone & $1(2.5 \%)$ & 0 & 0 & 0 & 0 & $1(2.5 \%)$ & $2(5 \%)$ \\
Intertrochanteric femoral & $1(2.5 \%)$ & $1(2.5 \%)$ & 0 & 0 & 0 & 0 & $2(5 \%)$ \\
Ankle & $2(5 \%)$ & 0 & 0 & 0 & 0 & 0 & $2(5 \%)$ \\
Total & $17(42.5 \%)$ & $14(35 \%)$ & $4(10 \%)$ & 0 & 0 & $5(12.5 \%)$ & $40(100 \%)$ \\
\hline
\end{tabular}

Table 3. LASSO regression analysis of PI risk related factors in the elderly inpatients with kidney disease.

\begin{tabular}{|c|c|c|}
\hline Variables & Assignment Meanings & $\begin{array}{c}\text { Variable } \\
\text { Coefficient } \beta\end{array}$ \\
\hline History of present respiratory infection/respiratory failure & 1: No, 2: Yes & 0.958 \\
\hline Craniocerebral diseases & 1: No, 2: Yes & 0.446 \\
\hline Limb mobility & 1: Independent, 2: Partial dependent, 3: Completely dependent & 0.387 \\
\hline Diabetes mellitus & 1: No, 2: Yes & 0.364 \\
\hline Hospitalization time & 1: $(>1), 2:(8 \sim), 3:(15 \sim), 4:(22 \sim), 5:(\geq 29)$ & 0.269 \\
\hline Edema & 1: No, 2: Yes & 0.273 \\
\hline Norton score & 1: 13 - 14 points (medium risk), $2: \leq 12$ points (high risk) & 0.266 \\
\hline Defecation & 1: Controllable, 2: occasionally incontinent, 3 : completely incontinent & 0.228 \\
\hline Hemoglobin & $\begin{array}{l}\text { 1: }>120(\text { male }) / 110 \text { (female) Normal, } 2:(90-120 \text { male/110 female) Mild; } \\
3:(60-90) \text { moderate, } 4:(30-59) \text { Severe, } 5:<30 \text { Very heavy }\end{array}$ & 0.136 \\
\hline Serum creatinine & Actual values & 0.093 \\
\hline Hypertension & $1=$ none, $2=$ yes & -0.301 \\
\hline Gender & $1=$ male, $2=$ female & -0.616 \\
\hline
\end{tabular}


related factors with PI risk were gender, hospitalization time, combined diseases (diabetes, hypertension, history of present respiratory infection/respiratory failure, craniocerebral disease, hemoglobin, creatinine, edema, Norton score, limb mobility, and defecation). Based on the GLMNET package of R software, influence factors were screened by LASSO regression. When lambda parameter was at the minimum value, i.e. lambda.min was $0.0169624,12$ influence factors were screened out, as shown in Table 4. In order to further verify, Logistic regression model was established, and the results showed 11 factors $(P>0.05)$ and 1 factor $(P>0.01)$. Then a standard deviation was added on the basis of lambda.min, and the optimal value of lambda.1se being 0.08248139 was selected, and influence factors were screened for the second time. The AUC value (Area Under Curve) and the optimal $\lambda$ value in the LASSO regression process are shown in Figure 1. The vertical coordinate represents the target parameter, the lower coordinate represents $\log (\lambda)$, and the upper horizontal coordinate is the number of non-zero coefficient variables in the model. For each value, a confidence interval of the target parameter can be obtained around the mean value of the target parameter shown by red dots. Two dashed lines in Figure 1 indicate two special $\lambda$ values, namely lambda.min and lambda.1se. Lambda.min is to get the $\lambda$ value of mean value of the minimum target parameter among all $\lambda$ values. Lambda.1se is the $\lambda$ value of the simplest model in a varying range of lambda.min. Figure 2 shows the LASSO coefficient profiles of the 17-texture feature. A coefficient profile plot is produced against the $\log (\lambda)$ sequence. Vertical line is drawn at the value selected using 10-fold cross-validation, where optimal $\lambda$ results in 2 nonzero coefficients.

Table 4. Logistic regression analysis results of PI risk factors for elderly kidney disease determined by lambda minimum.

\begin{tabular}{ccccc}
\hline Variables & $\begin{array}{c}\text { Regression } \\
\text { Coefficient }\end{array}$ & $\begin{array}{c}\text { Standard } \\
\text { Error }\end{array}$ & Wald Z & $P$ Value \\
\hline $\begin{array}{c}\text { History of present respiratory } \\
\text { infection/respiratory failure }\end{array}$ & 1.2454 & 0.5324 & 2.34 & 0.0193 \\
Craniocerebral diseases & 0.6661 & 0.5493 & 1.21 & 0.2253 \\
Limb mobility & 0.6613 & 0.5249 & 1.26 & 0.2077 \\
Diabetes mellitus & 0.8857 & 0.5805 & 1.53 & 0.1271 \\
Hospitalization time & 0.3967 & 0.2078 & 1.91 & 0.0563 \\
Edema & 0.6038 & 0.5374 & 1.12 & 0.2612 \\
Norton score & 0.4882 & 0.5517 & 0.88 & 0.3762 \\
Defecation & 0.3344 & 0.3567 & 0.94 & 0.3485 \\
Hemoglobin & -0.9994 & 0.5280 & -1.89 & 0.0584 \\
\hline Serum creatinine & 0.1805 & 0.1015 & 1.78 & 0.0753 \\
Hypertension & -1.1579 & 0.6631 & -1.75 & 0.0808 \\
Gender & 0.2749 & 0.85 & 0.3945
\end{tabular}


3) When selecting the optimal Lambda.1se value, in order to verify the results of LASSO variable selection, a Logistic regression model was established, which showed that its fitting and prediction were good. See Table 5.

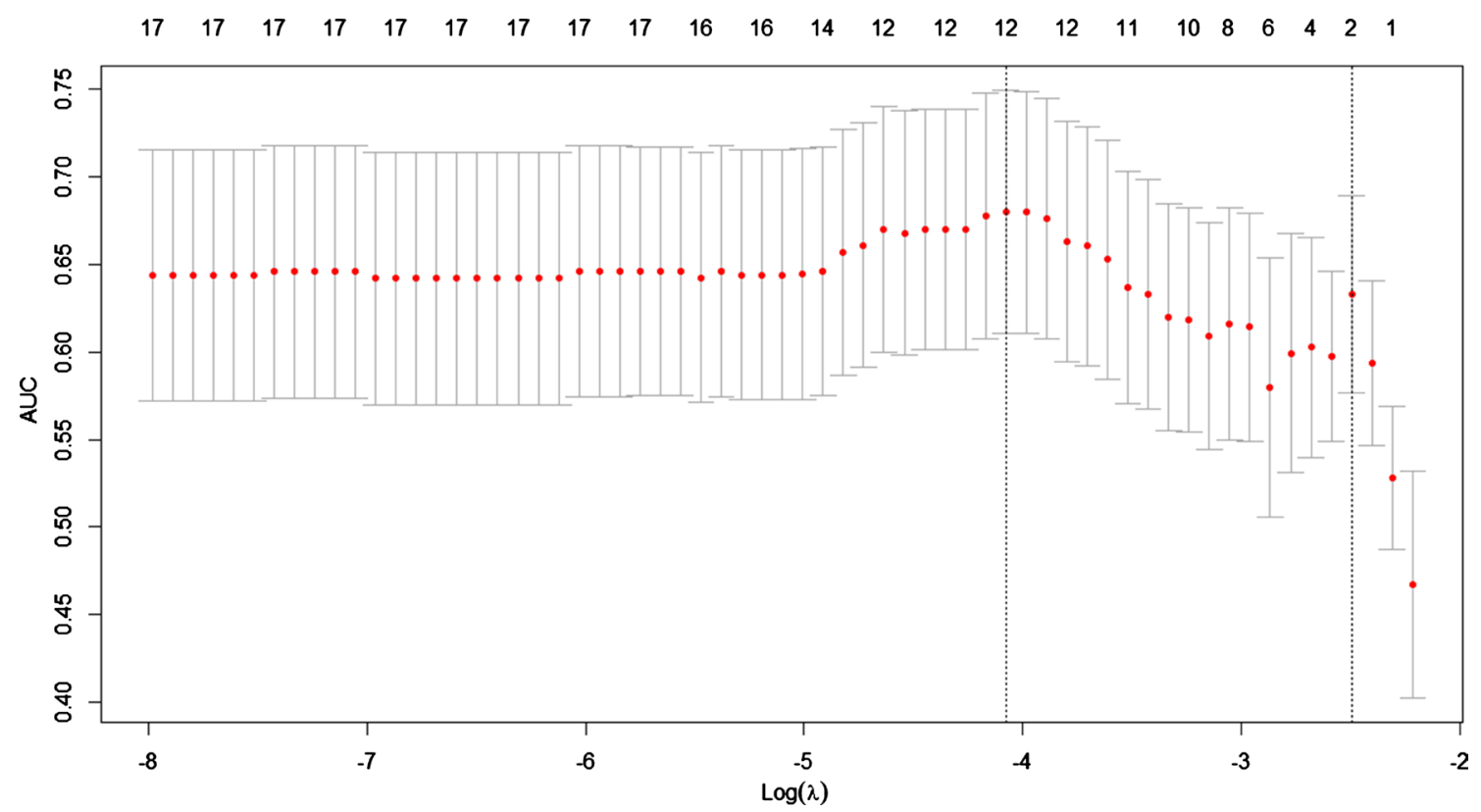

Figure 1. Texture feature selection.

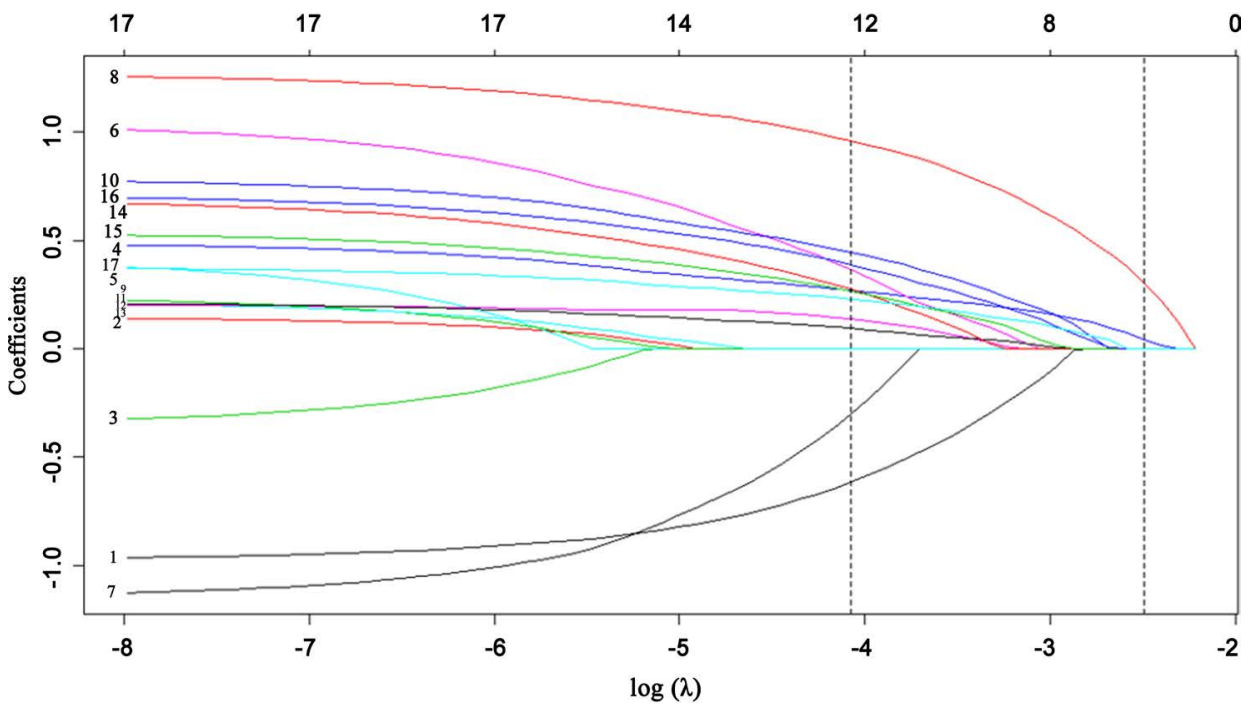

Figure 2. Lasso coefficient profiles.

Table 5. Results of Logistic regression analysis of PI risk factors for elderly kidney disease determined by lambda optimal value.

\begin{tabular}{ccccc}
\hline Variables & $\begin{array}{c}\text { Regression } \\
\text { Coefficient }\end{array}$ & $\begin{array}{c}\text { Standard } \\
\text { Error }\end{array}$ & Wald Z & $P$ Value \\
\hline $\begin{array}{c}\text { History of present respiratory } \\
\text { infection/respiratory failure }\end{array}$ & 1.2714 & 0.4572 & 2.78 & 0.0054 \\
Hospitalization time & 0.4177 & 0.1739 & 2.40 & 0.0163 \\
\hline
\end{tabular}




\section{Discussion}

\subsection{Significance of This Study on PI Risk in the Elderly Inpatients with Kidney Disease}

PI is often one of the common nursing problems in clinical departments. The occurrence of PI not only affects the state of illness, reduces the quality of life, increases the family burden and medical expenses, but also easily causes the patients' self-perceived burden (SPB), which brings physical and mental pain to patients, even threatens their life safety in serious situations [15] [16]. The incidence of hospital acquired PI is an indicator of nursing quality. How to avoid the occurrence of hospital acquired PI in the elderly patients with kidney disease is an important part of current nursing research. Most pressure injury can be prevented by effective nursing intervention [17]. PI evaluation is the basis of high-risk pressure injury management [18]. There are various influence factors of PI in the elderly inpatients, and different diseases and different individuals are different. Based on LASSO Logistic regression model, this study analyzes and screens the relevant factors of PI risk, aiming at timely intervening relevant factors, reducing the incidence of PI in the elderly inpatients with kidney disease, and improving their survival rate and quality of life.

\subsection{Characteristics of PI in the Elderly Inpatients with Kidney Disease}

Studies showed that the incidence of PI in the elderly patients has reached as high as $10 \%-25 \%$, and the mortality rate has increased by 6 times; the incidence of PI in inpatients has been $3 \% \sim 12 \%$ [19]. The results of this study showed that the proportion of hospital acquired PI in the elderly patients with kidney disease was $20.25 \%$, which was consistent with the percentage of PI incidence in the elderly patients, higher than the incidence of PI in inpatients. Researches by McAdams DeMarco et al. [20] show that compared with the ordinary elderly, the elderly patients with kidney disease are more likely to suffer from weakness, walking disorder, limited function and even severe disability. In addition, there are many combined diseases, complex etiology, diversified pathological changes, high incidence of end-stage renal disease (ESRD) in the elderly patients [4], so the probability of PI incidence increased [21]. Consistent with the results of this study, the elderly patients with kidney disease and PI risk are more likely to have hospital acquired PI. More attention should be paid to clinical nursing, and effective measures should be taken to reduce its incidence.

\subsection{Exploration on Influence Factors of Hospital Acquired PI in the Elderly Inpatients with Kidney Disease}

\subsubsection{The Incidence of PI in the Elderly Patients with Kidney Disease Affected by History of Present Respiratory Infection/Respiratory Failure}

When the elderly patients with kidney disease and medium \& high PI risk are complicated with respiratory infection or respiratory failure, they will have dif- 
ferent degrees of aeration (or) and ventilation dysfunction, resulting in the decrease or loss of activity tolerance, the increase of bed time, and even adoption of forced positions, such as raising bed head or even sitting position. PI is one of the common complications of long-term bedridden patients. The localized damage of skin (or) and subcutaneous tissue caused by pressure, shear force or friction often occurs at the bone protrusion [22]. Abroad researchers like Peterson et al. [23], used the whole-body pressure mapping to study and found that the sacral pressure was the lowest when bed head was raised $0^{\circ}$, while the pressure increased significantly when bed head was raised $30^{\circ}, 45^{\circ}, 60^{\circ}$ and $75^{\circ}$. The research results of Sun Yan et al. [24] [25] in China showed that the pressure on sacrococcygeal region was significantly higher in half lying position than in supine position. In this study, history of present respiratory infection/respiratory failure $(\beta=1.2714 . P<0.05)$, and the location of PI being sacrococcygeal $(60 \%)$ indicates that history of present respiratory infection/respiratory failure has a strong impact on the incidence of hospital acquired PI in the elderly inpatients with kidney disease, which means that the elderly inpatients with kidney disease carrying history of present respiratory infection/respiratory failure are more likely to have PI, and sacrococcygeal is the high-risk position where the elderly patients with kidney disease carry PI.

\subsubsection{The Incidence of PI Affected by Hospitalization Time of the Elderly Patients with Kidney Disease}

The elderly patients with kidney disease and medium \& high risk of PI are often accompanied by risk factors such as limited activity, long time in bed, decreased resistance, malnutrition caused by long-term consumption and so on. The longer the hospitalization time is, the risk of PI is higher. Some studies have shown that the risk of PI in patients staying in hospital for 11 - 25 days, 26 - 40 days and more than 40 days is $2.849,5.549$ and 37.260 times respectively higher than that in patients staying in hospital for less than 11 days; The risk of PI increased significantly with the increasing length of stay, especially in patients staying in hospital for over 40 days [26]. In this study, hospitalization time $(\beta=0.4177$. $P<$ 0.05 ) also confirms that the incidence of hospital acquired PI in the elderly patients with kidney disease and PI risk was related to hospitalization time.

\subsection{Clinical Implications}

In this study, LASSO Logistic regression model was used to screen variables, and then the relevant influence factors were analyzed. History of present respiratory infection/respiratory failure and hospitalization time were independent factors of hospital acquired PI risk in the elderly inpatients with kidney disease. In the prevention and treatment of the elderly patients with kidney disease and medium \& high PI risk, for patients with respiratory infection and respiratory failure, the nursing staff should timely and accurately assess PI risk while following the doctor's instructions to treat the patients with cough, asthma and anti-infection. In clinical nursing work, we can improve respiratory symptoms and 
increase patients' activity tolerance by strengthening ward management, implementing basic nursing, monitoring changes of vital signs, doing well in psychological nursing and health education, and guiding respiratory function exercise, etc. For patients with long hospital stay, we should do a regular and accurate PI risk assessment. In clinical work, we should do "seven things frequently", that is, observe frequently, turn over frequently, massage frequently, scrub frequently, organize frequently, change frequently and hand over frequently. If necessary, PI prevention materials and appliances should be used in combination with clinical practice. In addition, we should actively deal with the primary disease, promote the early recovery of patients, and reduce the incidence of PI in the elderly inpatients with kidney disease.

\section{Conclusion}

The elderly inpatients with kidney disease are the high-risk group of hospital acquired PI. The body part with high incidence of PI is sacrococcygeal, and the most common injury is stage 1 . History of present respiratory infection or respiratory failure and hospitalization time are independent factors of hospital acquired PI risk in the elderly patients with kidney disease, among which history of present respiratory infection or respiratory failure is an important influence factor of hospital acquired PI in elderly patients with kidney disease; and there is a positive correlation between hospitalization time and the incidence of PI in elderly patients with kidney disease. This provides a reference for improving preventive measures and reducing the incidence of PI in clinical nursing. The sample size in this study is small. In follow-up studies, in order to further improve and perfect the results in this study, the sample size should be enlarged; more possible influence factors should be included; each sub-factor should be subdivided layer by layer, and other prediction tools based on other mathematical models should be built.

\section{Acknowledgements}

This study was supported by grants from Guangdong Medical Science and Technology Research Foundation (Grant NO. A2020362, A2019120), and special funds for nursing research from The First Clinical Medical College of Jinan University (Grant NO. 2014311).

\section{Conflicts of Interest}

The authors declare no conflicts of interest regarding the publication of this paper.

\section{References}

[1] National Pressure Ulcer Advisory Panel (NPUAP) Announces a Change in Terminology from Pressure Ulcer to Pressure Injury and Updates the Stages of Pressure injury, 2017-06-05. 
[2] Jin, X.Y. and Xie, L.F. (2014) Evaluation, Prevention and Treatment of Pressure Injury. Chinese Journal of Injury and Repair (Electronic Edition), 9, 189-194. (In Chinese)

[3] Khor, H.M., Tan, J., Saedon, N.I., et al. (2014) Determinants of Mortality among Older Adults with Pressure Ulcers. Archives of Gerontology and Geriatrics, 59, 536-541. https://doi.org/10.1016/j.archger.2014.07.011

[4] Ren, J.W., Zhang, X.M., Dai, H.Z., Zhang, J.G., Li, K.L., He, Y.N. and Lin, L.R. (2018) Analysis of Clinical and Pathological Characteristics of the Elderly Patients with Kidney Disease. Chinese Journal of Nephrology, 7, 163-166. (In Chinese)

[5] Liu, H. and Fu, X.Y. (2007) Research Progress on Risk Factors Assessment and Prevention of Pressure Injury. China Nursing Management, 7, 50-51. (In Chinese)

[6] Yao, L. (2016) Practical Manual of Nursing Sensitive Quality Indicators. People's Health Press, Beijing, 115-120. (In Chinese)

[7] Bergstrom, N., Braden, B.J., Laguzza, A., et al. (1987) The Braden Scale for Predicting Pressure Injury Risk. Nursing Research, 36, 205-210.

https://doi.org/10.1097/00006199-198707000-00002

[8] Norton, D. (1989) Calculating the Risk: Reflections on the Norton Scale. Decubitus, 2, 24-31.

[9] Rabinovitz, E., Finkelstein, A., Ben Assa, E., et al. (2016) Norton Scale for Predicting Prognosis in the Elderly Patients Undergoing Trans-Catheter Aortic Valve Implantation: A Historical Prospective Study. Journal of Cardiology, 67, 519-525. https://doi.org/10.1016/j.jjcc.2016.01.017

[10] Jin, Z. and Zhang, F.C. (2011) Application of Norton Pressure Injury Evaluation Scale in the Prevention of Pressure Injury. Nursing Practice and Research, 8, 87-88. (In Chinese)

[11] Tibshirani, R. (1996) Regression Shrinkage and Selection via the LASSO. Journal of the Royal Statistical Society, Series B, 58, 267-288. https://doi.org/10.1111/j.2517-6161.1996.tb02080.x

[12] Cheng, J., Liang, X., Zheng, S.S., Wang, J., Ding, L.J., Wang, Y. and Lu, W.L. (2018) A Study on the Screening and Utilization of High-Risk Breast Cancer Population Based on LASSO Logistic Regression Model. Chinese Journal of Disease Control, 22, 551-554 + 559. (In Chinese)

[13] Archer, K.J. and Williams, A.A. (2012) L1 Penalized Continuation Ratio Models for Ordinal Response Prediction Using High-Dimensional Datasets. Statistics in Medicine, 31, 1464-1474. https://doi.org/10.1002/sim.4484

[14] Yu, L.M., Wu, W., Sun, G.Z., Wang, J.Q., et al. (2006) Internal Medicine Nursing. 4th Edition, People's Health Publishing House, Beijing, 8. (In Chinese)

[15] Cousineau, N., McDowell, I., Hotz, S., et al. (2003) Measuring Chronic Patients' Feelings of Being a Burden to Their Caregivers Development and Preliminary Validity on of a Scale. Medical Care, 41, 110-118. https://doi.org/10.1097/00005650-200301000-00013

[16] Ding, Y.M. and Wang, W. (2013) China Pressure Sore Care Guidance. Stoma, Wound, Incontinence Care Professional Committee of China Nursing Society, Beijing, 45. (In Chinese)

[17] Carlsson, M. and Gunningberg, L. (2017) Unavoidable Pressure Ulcers at the End of Life and Nurse Understanding. British Journal of Nursing, 26, S6-S17. https://doi.org/10.12968/bjon.2017.26.Sup20.S6

[18] Krupp, A.E. and Monfre, J. (2015) Pressure Ulcers in the ICU Patient: An Update 
on Prevention and Treatment. Current Infectious Disease Reports, 17, 1-6. https://doi.org/10.1007/s11908-015-0468-7

[19] Jiang, Q.X., Guan, X.P., Su, C.Y., Guo, X.J., Chen, Y.J., Zhu, Y.J., Jia, J., Bai, S.P., Zhang, R., Liu, L., Xu, L., Wu, L.Y. and Liu, Y. (2013) Multi-Center Joint Survey on the Prevalence of Pressure Injury in General Hospitals. China Nursing Management, 13, 26-30. (In Chinese)

[20] Mcadam-Demarco, M.A., Law, A., Salter, M.L., et al. (2013) Frailty as a Novel Redirector of Mortality and Hospitalization in Individuals of Ages Undergoing Hemodialysis. Journal of the American Geriatrics Society, 61, 896-901. https://doi.org/10.1111/jgs.12266

[21] Wang, J. (2019) Clinical Nursing Analysis of Patients with Chronic Kidney Disease Complicated with Pressure Injury. Electronic Journal of Practical Clinical Nursing, 4, 196-197. (In Chinese)

[22] National Pressure Ulcer Advisory Panel and the European Pressure Ulcer Advisory Panel (NPAP/EPUAP) (2009) Prevention and Treatment of Pressure Ulcers: Clinical Practice Guideline. NPUAP, Washington DC.

[23] Peterson, M., Schwab, W., McCutcheon, K., et al. (2008) Effects of Elevating the Head of Bed on Interface Pressure in Volunteers. Critical Care Medicine, 36, 3038-3042. https://doi.org/10.1097/CCM.0b013e31818b8dbd

[24] Sun, Y., Cui, F.F., Zhang, L., et al. (2013) Effects of Local Continuous Compression on Skin Blood Perfusion and Temperature in Different Postures. Chinese Journal of Burn, 29, 67-69. (In Chinese)

[25] Yang, X, Cao, W., Weng, C.X., et al. (2011) Analysis of the Characteristics and Influence Factors of Local Body Surface Pressure in Four Kinds of Recumbent Positions. Journal of Nursing, 26, 7-9. (In Chinese)

[26] Duan, Z.Z. and Liu, Y.L. (2012) Case Control Study on Risk Factors of Pressure Injury in ICU Patients. Journal of Nursing, 27, 42-44. (In Chinese) 